IN PRACTICE

\title{
Comparison of non-invasive sampling methods for detection of HPV in rural African women
}

\author{
N Lack, B West, D Jeffries, G Ekpo, L Morison, W P Soufter, G Walraven, L Boryseiwicz
}

Sex Transm Infect 2005;81:239-241. doi: 10.1136/sti.2004.010413

Background: The prevalence of cervical cancer is extremely high in low income countries, primarily because of a lack of cytological screening. The link between human papillomavirus (HPV) and cervical cancer has long been recognised, and it has been suggested that isolated HPV testing in women who do not participate in existing screening programmes may be used to identify women at higher risk of developing cervical cancer. This community based study compares two self administered techniques for detecting HPV (tampons and self administered swabs) with a clinician directed technique, the cervical cytobrush.

Methods: 377 rural women were interviewed and of these 210 women had full gynaecological examination, and accepted all three sampling methods for HPV. HPV typing of DNA extracts was performed using polymerase chain reaction and enzyme linked immunosorbent assay techniques.

Results: Using the cervical cytobrush as the gold standard, self administered swabs (SAS) showed a sensitivity of $63.9 \%$, and tampons showed a sensitivity of $72.4 \%$. The acceptability of these two tests was $97.1 \%$ and $84.6 \%$ respectively. When combining acceptability with sensitivity, the SAS detected $61.9 \%$ and the tampons detected $60.9 \%$ of the true positives. Conclusion: In a setting where women are at a considerable risk of developing cervical cancer, with no access to a formal screening programme, self directed HPV testing could be a useful screening tool in identifying those women at increased risk who may require further investigation.

\footnotetext{
C
} ervical cancer affects approximately 500000 women each year ${ }^{1} ; 80 \%$ of these cases occur in the developing world where it is by far the commonest cancer in women. $^{2}$ Here, morbidity and mortality are high because cytological screening is not readily available and women tend to present late with advanced stage disease when treatment options are limited.

The link between human papillomavirus (HPV) and cervical cancer has long been recognised ${ }^{134}$ and the detection of HPV has been used as an adjunct to cervical cytology to identify women who are at risk of developing cervical cancer. ${ }^{56}$ Although it is recognised that this is the best use of HPV testing, isolated HPV testing in women who do not participate in existing screening programmes may be useful. ${ }^{7}$

Now that HPV vaccines are being developed and tested, there is a need to test for HPV in low income counties, in both the field and clinic settings. The purpose of this study is to compare the acceptability and sensitivity of two different types of non-invasive techniques for detecting HPV (self administered swabs and tampons) with a clinician directed technique, the cervical cytobrush.

\section{METHODS}

Between February and June 2002, women who had previously been screened for HPV in a reproductive morbidity survey (RMS) ${ }^{8}$ were recruited from a rural area of the Gambia All women from the RMS who had previously accepted a speculum examination were invited to participate in the present study. Those who were pregnant at the time of interview were excluded from the study.

Women were interviewed by a female gynaecologist, and were then verbally instructed to collect a vaginal sample using a sterile self administered Dacron swab (SAS) inserted high into the vagina and rotated five times, witnessed by the doctor. The sample was then placed in phosphate buffered saline and stored in a cool box until the end of the day when it was frozen and stored at $-70^{\circ} \mathrm{C}$. Cervical smears were taken, and HPV sampling was performed by inserting a cytobrush into the cervix and rotating it through $360^{\circ}$. This was placed immediately in $1 \mathrm{ml}$ of phosphate buffered saline, kept in a cool box until the end of the day, then stored at $-70^{\circ} \mathrm{C}$. Finally, the participants were asked to insert a tampon into the vagina, which was removed 1 hour later and stored in a cool box until the end of the day when the contents of the tampon were then expelled into phosphate buffered saline and stored at $-70^{\circ} \mathrm{C}$. Women were examined and treated for reproductive tract infections in agreement with WHO syndromic management guidelines. Cervical smears were assessed at the Department of Pathology, Llandough Hospital, Cardiff, UK, and were classified by the Bethesda system. Those women with abnormal cytology were offered treatment.

DNA was extracted from samples using QIAmp DNA minikit (Quiagen Ltd, Crawley, UK) and HPV polymerase chain reaction (PCR) was performed as described previously. Specimens were HPV typed using enzyme linked immunosorbent assay (ELISA) and monospecific probes.

\section{RESULTS}

In all, 377 of the 946 eligible women attended for interview $(39.9 \%)$. Table I shows the proportions of women accepting each test; 224 women accepted all three sampling methods $(59.4 \%)$, and of these, 210 were $\beta$ globin positive or HPV positive and can be used for comparison $(55.7 \%)$.

$\chi^{2}$ Tests showed that there was no significant difference in the proportions with DNA detected, or in the prevalence of HPV between the three sampling methods.

Of the 210 women for whom HPV results were available in all three samples, 36 were positive for HPV in the cervical cytobrush specimen. Using this as the gold standard, the tampons had a sensitivity of $72.2 \%$ (95\% CI $57.6 \%$ to $86.8 \%$ ) and a specificity of $92.5 \%$, with a positive predictive value of $66.7 \%$ and a negative predictive value of $94 \%$; the SAS had a

Abbreviations: ELISA, enzyme linked immunosorbent assay; HPV, human papillomavirus; PCR, polymerase chain reaction; RMS reproductive morbidity survey; SAS, self administered swabs 
Table 1 Acceptability of each test, efficiency at collecting DNA, and prevalence of HPV

\begin{tabular}{|c|c|c|c|c|c|}
\hline Sampling method & $\begin{array}{l}\text { \% Accepting test } \\
\text { (number accepting } \\
\text { out of } 377)\left(n^{*}\right)\end{array}$ & $\begin{array}{l}\% \text { with } \\
\text { DNA }\left(n^{*}\right)\end{array}$ & $\begin{array}{l}\text { \% with } \\
\text { HPV }\left(n^{\star}\right)\end{array}$ & $\begin{array}{l}\text { Sensitivity } \\
\text { of test }\end{array}$ & $\begin{array}{l}\text { \% True positives } \\
\text { detected adjusting } \\
\text { for acceptability }\end{array}$ \\
\hline Cervical brush & $72.4 \%(273)$ & 97.9 (273) & $15.3(268)$ & 100 & 72.4 \\
\hline Tampon & $84.4 \%(318)$ & $97.4(318)$ & $17.4(310)$ & 72.2 & 60.9 \\
\hline $\begin{array}{l}\text { Self administered } \\
\text { swab }\end{array}$ & $97.1 \%(366)$ & $98.9(366)$ & $16.3(362)$ & 63.7 & 61.9 \\
\hline $\begin{array}{l}\text { Tampon + self } \\
\text { administered swab }\end{array}$ & $82.5 \%(311)$ & $96.1(299)$ & $22.8(298) \dagger$ & 77.8 & 64.2 \\
\hline
\end{tabular}

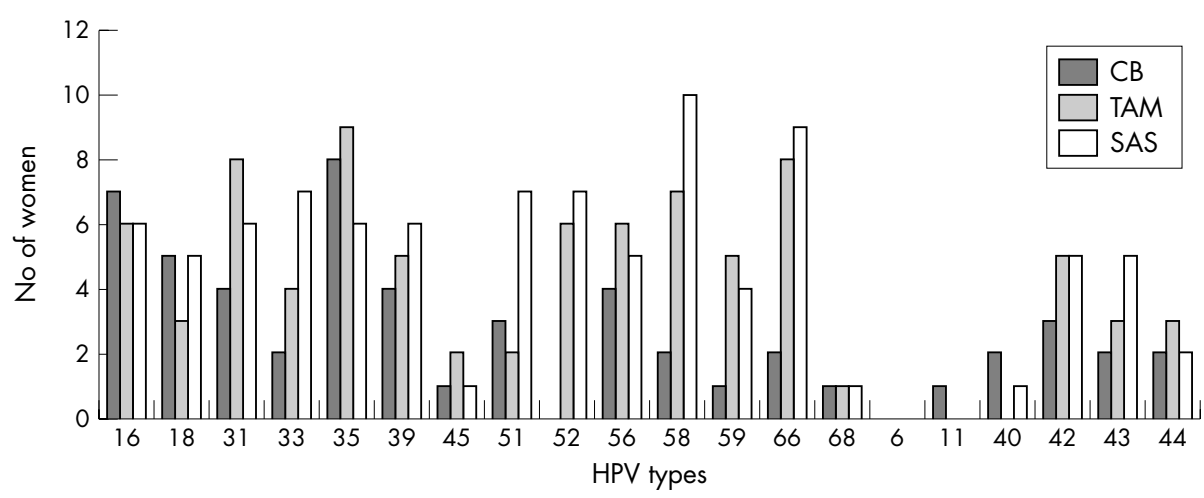

Figure 1 HPV types in the three different sampling methods. $C B$, cytobrush; TAM, tampon; SAS, self administered swabs.

sensitivity of $63.9 \%$ (51.9\% to $83.3 \%)$, and a specificity of $93.7 \%$, with a positive predictive value of $67.6 \%$ and a negative predictive value of $92.6 \%$.

When acceptability of the sampling method was combined with the sensitivity of the test, the self administered tampon and swab detected $60.9 \%$ and $61.9 \%$ of the true positives, respectively, compared with $72.4 \%$ by the cervical cytobrush method (table 1).

If the SAS and tampon tests are combined, such that the result is considered positive if either test is positive, then compared to SAS alone, the combined test shows a significant increase in sensitivity (with cervical brushes as the gold standard) of $14 \%(3 \%$ to $25 \%)$ and a significant decrease in specificity of $5 \%$ ( $3 \%$ to $9 \%)$. However, once acceptability has been taken into account, there is little gain in the proportion of true positives detected by combining the two tests (table 1 ).

For all three sampling methods, high risk HPV types were more prevalent in our sample, than low risk types. Figure 1 shows the distribution of HPV types in this population, using the three different sampling methods.

\section{DISCUSSION}

The study described in this paper is one of the very rare community studies in a selected population of women based in a country that is unable to offer a formal screening programme for cervical cancer. In such low income countries where health infrastructure is poor, HPV testing has the potential to be a valuable tool for the prevention of cervical cancer.

Previous studies have looked at the possibility of different methods of sampling cervical DNA in order to diagnose both cytological abnormalities and those women who are at higher risk of cervical cancer-those with persistent high risk HPV infection. ${ }^{10}$

Several studies have shown that clinician directed testing for HPV has a sensitivity for detection of high grade lesions or cervical cancer that is equivalent or superior to that of cytology. Ogilvie et al (Diagnostic accuracy of self obtained
HPV cervical-vaginal samples vs clinician obtained samples: a systematic review, 2003: unpublished) reviewed the diagnostic accuracy of self obtained HPV cervicovaginal samples verses clinician obtained samples. They found that in all studies reviewed, the sensitivity and specificity were diminished when patients collected their own samples. This was consistent with our findings. The sensitivity of the SAS in our population was $63.7 \%$, which is slightly lower than in other similar studies; however, it is important to note that in previous studies women were often recruited from colposcopy or genitourinary medicine clinics, or have known cervical cancer. These women are likely to have a higher HPV viral load in their cervico-vaginal secretions, which is easier to detect in self collected samples.

Studies of SAS and tampons performed in industrialised countries takes place in a society where tampon use is common. ${ }^{11}$ This is not the case in the Gambia, and little has been done to assess the acceptability of different types of sampling in sub-Saharan Africa. While less than half of the invited women took part in our study, SAS was highly acceptable among those who did. The acceptability of tampons was slightly lower, but still higher than for the clinician directed cervical cytobrush. Among participating women, each of the self directed methods detected approximately $60 \%$ of true positives. This would identify a substantial proportion of women who may be at higher risk of developing precancerous or cancerous change in the cervix. Further research is needed to explore how a screening programme based on HPV detection could operate within low income countries with poor health infrastructure. Further socioeconomic analysis is required to assess the effectiveness of self directed HPV testing as a screening strategy.

\section{ACKNOWLEDGEMENTS}

We thank Dr R L Bailey for critical review of this paper. We also thank all the field staff and laboratory assistants who contributed to the study, represented by Louis Loppy and Nkaye Kanyi; Maimuna Sowe for data management; Department of Cytology and 
Histopathology, Llandough Hospital, Cardiff and Vale NHS Trust for cervical cytology and histology analysis.

\section{CONTRIBUTORS}

LB, GW, PS, LM, and BW were responsible for study design, and NL carried out field work, gynaecological examinations, laboratory analysis (including HPV testing and typing) and drafting of the paper; BW was also responsible for supervision of laboratory work; GE was involved in field work and was also one of the two gynaecologists on the study; DJ and LM performed the statistical analysis and, with PS, were responsible for interpretation of results; all authors were involved in reviewing drafts of the paper, and contributed to the final version.

\section{Authors' affiliations}

N Lack, B West, D Jeffries, G Ekpo, G Walraven, MRC Laboratories, Fajara, PO Box 273, Banjul, Gambia

W P Soutter, L Boryseiwicz, Imperial College, London SW7 2AZ, UK L Morison, London School of Hygiene and Tropical Medicine, London, UK

This work was supported by funding from the Medical Research Council (UK).

Correspondence to: Dr Nicola Lack, MRC Laboratories Fajara, Atlantic Boulevard, PO Box 273, Banjul, Gambia; nikkilack@hotmail.com

Accepted for publication 21 September 2004

\section{REFERENCES}

1 Bosch FX, Manos MM, Munoz N, et al. Prevelance of human papillomavirus in cervical cancer: a worldwide perspective. J Natl Cancer Inst 1995:87:796-802.

2 Parkin D M, Pisani P, Ferlay J, et al. Estimates of the worldwide incidence of 25 cancers in 1990. Int J Cancer 1999;80:827-41.

3 Zur Hausen H. Condylomata acuminata and human genital cancer. Cancer Res, 1976;36:794.

4 Walboomers JMM, Jacobs MV, Manos MM, et al. HPV is a necessary cause of invasive cervical cancer worldwide. J Pathol 1999;189:12-19.

5 Cuzick J, Szarewski A, Terry G, et al. Human papillomavirus testing in primary cervical screening. Lancet 1995;345:1533-66.

6 Nobbenhuis MAE, Walboomers JMM, Helmerhorst TJM, et al. Relation of human papillomavirus status to cervical lesions and consequences for cervical cancer screening: a prospective study. Lancet 1999;354:20-25.

7 Cuzick J, Szarewski A, Cubie H, et al. Management of women who test positive for high-risk types of human papillomavirus: the HART study. Lancet 2003;362:1871-6.

8 Walraven G, Scherf C, West B, et al. The burden of reproductive-organ disease in rural women in the Gambia, West Africa. Lancet 2001;357:1161-7.

9 Jacobs MV, Snijders PJF, van den Brule AJC, et al. A general primer GP5+/ GP6+ -mediated PCR-enzyme immunoassay method for rapid detection of 14 high-risk and 6 low-risk human papillomavirus genotypes in cervical scrapings. J Clin Microbiology 1997;35:791-5.

10 Salmeron J, Lazcano-Ponce E, Lorincz A, et al. Comparison of HPV-based assays with Papanicolaou smears for cervical cancer screening in Morelos State, Mexico. Cancer Causes Control 2003;14:505-12.

11 Harper DM, Noll WW, Belloni DR, et al. Randomised clinical trial of PCRdetermined human papillomavirus detection methods: self-sampling versus clinician-directed-biologic concordance and women's preferences. Am J Obstet Gynaecol 2002; 186:365-73.

\section{Call for papers}

11 th European Forum on Quality Improvement in Health Care 26-28 April 2006, Prague, Czech Republic

Deadline 30 September 2005.

For further information and to submit online go to: www.quality.bmipg.com 